\title{
Radio observations of SN 1006
}

\author{
Estela M. Reynoso $\dagger$ \\ Instituto de Astronomía y Física del Espacio, C.C. 67, Suc. 28, 1428 Buenos Aires, Argentina \\ email: ereynoso@iafe.uba.ar
}

\begin{abstract}
In this article I review our present knowledge of SN 1006 based on radio observations since the identification of the radio remnant four decades ago. I also report preliminary results of a new radio expansion study which combines VLA 1991/92 data with new VLA and ATCA observations performed in 2003.
\end{abstract}

Keywords. (ISM:) supernova remnants, individual: SN 1006, radio continuum: ISM, radio lines: ISM, polarization.

Based on a polarization of about $10 \%$ and a non-thermal spectral index of -0.6 , Gardner \& Milne (1965) concluded that the catalogued radio source PKS 1459-41 was the remnant of the SN recorded in 1006. SN 1006, the faintest of the historical SNRs in radio wavelengths, depicts a $\sim 30^{\prime}$ diameter shell with a remarkable bilateral symmetry, where the symmetry axis is perpendicular to the Galactic plane. Estimates of the distance to SN 1006 based on different arguments yield values varying approximately from 1.4 to $2.2 \mathrm{kpc}$. Some correspondence between the SNR and H f features suggest a systemic velocity of $\sim-20 \mathrm{~km} \mathrm{~s}^{-1}$, which corresponds to a distance of $1.7 \mathrm{kpc}$ (Dubner et al. 2002). High-resolution polarization maps of SN 1006 (Reynolds \& Gilmore 1993) show a radial distribution of the magnetic field, typical of young SNRs, with a mean polarization of $13 \%$ and peaks of $30 \%$.

The expansion of the radio remnant of SN 1006 was measured by Moffett, Goss \& Reynolds (1993), comparing two VLA (NRAO) images obtained within an interval of $\sim 8$ yr. They obtained a mean expansion parameter $m=0.48 \pm 0.13$, where $m$ is defined as $R \propto t^{m}$. A new radio expansion study is currently being carried out based on archival 1991/92 VLA data and new observations performed simultaneously in 2003 with the ATCA (Australia) and the VLA. We obtain an average $m=0.53 \pm 0.40$, with pronounced azimuthal variations. The brightest lobes are expanding much faster than the faintest regions, with $m$ typically between 0.6 and 0.8 . Such values are compatible with a remnant in which the ejecta, with density described by an exponential or a $r^{-7}$ power law, expand into a circumstellar density profile of $r^{-2}$ (Dwarkadas \& Chevalier 1998). Alternatively, the ambient density could be constant if the SNR is still in the ejecta-dominated phase. The velocities inferred for the shock front are in excellent agreement with recent numerical simulations (Velázquez, private communication).

\section{References}

Dubner, G. M., Giacani, E. B., Goss, W. M., Green, A. J., \& Nyman, L.-Å. 2002, A\&A, 387, 1047

Dwarkadas, V. V., \& Chevalier, R. A. 1998, ApJ, 497, 807

Gardner, F. F., \& Milne, D. K. 1965, AJ, 70, 754

Moffett, D. A., Goss, W. M., \& Reynolds, S. P. 1993, AJ, 106, 1566

Reynolds, S. P., \& Gilmore, D. M. 1993, AJ, 106, 272

$\dagger$ Member of the Carrera del Investigador Científico of CONICET, Argentina. 